\title{
AVALIAÇÃO DA HOMOGENEIDADE ESTRUTURAL EM LIGA DE ALUMÍNIO 7475-T735 I SUBMETIDA A LAMINAÇÃO CONVENCIONAL E ASSIMÉTRICA
}

\author{
Andrey de Moraes Barcelos Casanova ' \\ Saulo Brinco Diniz ${ }^{1,2}$ \\ Carolline Serafim da Silva ${ }^{3}$ \\ Talita Gama de Sousa ' \\ Angelo Siqueira da Silva ' \\ Luiz Paulo Mendonça Brandão ${ }^{4}$ \\ Andersan dos Santos Paula ${ }^{4}$
}

\section{Resumo}

Ligas de alumínio são amplamente empregadas no setor aeronáutico devido suas boas propriedades físico-químicas, onde estudos contínuos são desenvolvidos a fim de otimizar propriedades adequadas para processos de conformação diferenciados, a citar a conformação superplástica que o objetiva materiais de grão ultrafinos. Este trabalho teve por objetivo avaliar o potencial de promover assimetria microestrutural e mecânica em uma liga 7475-T735 I laminada a quente quando do processamento via laminação assimétrica com razão entre cilindro de I,67 em comparação com a laminação convencional a temperatura ambiente. No entanto, observou um resultado contraditório, pois a laminação assimétrica adotada promoveu um maior incremento, ao longo da espessura, na homogeneidade microestrutural e endurecimento quando comparada a laminação convencional.

Palavras-chave: Laminação convencional; Laminação assimétrica; DPS.

\section{EVALUTION OF THE STRUCTURAL HOMOGENEITY IN 7475-T735 I ALUMINUM ALLOY SUBMITTED TO CONVENTIONAL AND ASYMMETRIC ROLLING}

\begin{abstract}
Aluminum alloys are widely used in the aeronautical sector due to their good physicochemical properties. Continuous studies are developed in order to optimize suitable properties for differentiated conformation processes, such as the superplastic conformation that targets the ultrafine grain materials. This work aimed to evaluate the potential to promote microstructural and mechanical asymmetry in a 7475-T735 I hot rolled alloy when processing through asymmetric rolling with cylinder ratio of 1.67 compared to conventional rolling at room temperature. However, it observed a contradictory result, since the asymmetric rolling promoted a greater increase, along the thickness, in the microstructural homogeneity and hardening when compared to the conventional rolling.
\end{abstract}

Keywords: Conventional rolling; Asymmetric rolling; SPD.

\footnotetext{
'Programa de Pós-graduação em Ciência dos Materiais - PGCM, Instituto Militar de Engenharia - IME, Rio de Janeiro, RJ, Brasil. E-mail: andreymbc@hotmail.com

${ }^{2}$ Centro Federal de Educação Tecnológica Celso Suckow da Fonseca - CEFET, Angra dos Reis, RJ, Brasil.

${ }^{3}$ Galvasud, Porto Real, RJ, Brasil.

${ }^{4}$ Departamento de Ciência e Tecnologia, Instituto Militar de Engenharia - IME, Rio de Janeiro, RJ, Brasil.

*Versão revisada de trabalho apresentado na ABM Week 20I7, de 2 a 6 de outubro de 20I7, São Paulo, SP, Brasil.
}

2176-1523 (C) 2017 Associação Brasileira de Metalurgia, Materiais e Mineração. Publicado pela ABM. Este é um artigo de acesso aberto distribuído sob os termos da licença Creative Commons CC BY-NC-ND (Attribution-NonCommercial-NoDerivs) - https:// creativecommons.org/licenses/by-nc-nd/4.0\%. 


\section{INTRODUÇÃO}

A liga 7475 é uma variação da 7075 , onde foi desenvolvida visando uma melhora nas aplicações em que se requer maior resistência mecânica, à fratura, propagação de trinca e corrosão. Para tal, foi necessária a redução de elementos como ferro e silício da liga original assim como alteração do processo de envelhecimento. Desta forma, a liga 7475 possui grãos mais finos e é amplamente empregada para conformação de componentes de alto desempenho aeronáutico [I]. No entanto, o refinamento microestrutural obtido ainda põe limitações quando uso em processos de conformação superplástica e aplicações que exige estabilidade microestrutural mais alta temperatura.

As técnicas de deformação plástica severa (DPS) são capazes de introduzir ao material características físicas únicas, como refinamento de grãos, podendo assim, ter o material, a possibilidade de processamentos futuros para conformação superplástica. Dentre as técnicas de DPS, está a laminação assimétrica (LA), a qual tem grande potencial para aplicação industrial. Esta se caracteriza como uma técnica de laminação em que os dois cilindros utilizados podem trabalhar com velocidades de rotação diferentes, diâmetros destes, coeficientes de atritos distintos entre as superfícies de contato de cada um dos cilindros com as superfícies da chapa, ou até mesmo por fim a combinação dessas condições. Tal fato, irá realizar uma associação de compressão direta e de cisalhamento na chapa, podendo assim, ocorrer a redução dos tamanhos de grãos presentes na microestrutura $[2,3]$.

Devido a carência de informações na literatura relativas a liga 7475 na condição de tratamento térmico de superenvelhecimento associada a processos de conformação convencional ou técnicas de DPS, foi então motivado o presente estudo com enfoque na evolução microestrutural e mecânica da liga 7475 , submetida a dois diferentes processamentos de laminação (convencional e assimétrica) quando comparada com a condição de partida. As avaliações foram conduzidas com uso das técnicas de microscopia eletrônica de varredura (MEV) e ultramicrodureza instrumentada.

\section{MATERIAL E MÉTODOS}

O material de estudo foi proveniente de chapa laminada a quente de uma liga de alumínio 7475 (Tabela I), que inicialmente foi recebida com espessura de $12,7 \mathrm{~mm}$, fornecida na condição de tratamento T735I, ou seja, submetida a tratamento de solubilização, com posterior alívio de tensões por estiramento e endireitamento associado

Tabela I. Composição química da liga em estudo, percentual em massa [4]

\begin{tabular}{ccccccc}
\hline Al & $\mathbf{Z n}$ & $\mathbf{M g}$ & $\mathbf{C u}$ & $\mathbf{F e}$ & $\mathbf{S i}$ & Outros \\
\hline 89,5000 & 5,6700 & 2,3800 & 1,7800 & 0,2420 & 0,0438 & 0,3842 \\
\hline
\end{tabular}

com superenvelhecimento final, o que acarreta em uma redução na resistência mecânica, porém, visando o aumento da resistência a corrosão do material [5].

A liga na condição como recebida (CR) foi submetida a duas rotas distintas de laminação com uso de um laminador piloto, onde em ambas, objetivou-se espessura final de $3 \mathrm{~mm}$. Para a laminação convencional (LC), após I 3 passes foi obtida uma deformação acumulada verdadeira de $75 \%$. Para a laminação assimétrica, com uso de cilindros de distintos diâmetros (54,8 e 32,8 mm) com razão de assimetria de 1,67 , ao final de 18 passes obteve-se uma deformação acumulada verdadeira de $76 \%$.

As amostras, após corte na direção de laminação para análise ao longo da espessura, foram lixadas e polidas com pasta de diamante com granulometria de $6 \mu \mathrm{m}, 3 \mu \mathrm{m}$ e I $\mu \mathrm{m}$ para análise de ultramicrodureza. Para realizar análise no MEV, foi realizado polimento com alumina seguido de polimento eletrolítico com solução $30 \%$ ácido nítrico e $70 \%$ de metanol, pré resfriada em nitrogênio líquido.

As análises foram conduzidas um MEV, modelo Quanta 250 FEG - fabricante FEI, e um ultramicrodurômetro instrumentado, marca Shimadzu - modelo DUH-2I IS, com indentador Vickers. Todas as medidas de ultramicrodureza foram realizadas com carga mínima de 0,2 gf e carga máxima $\left(F_{\text {máx }}\right)$ de 50 gf e velocidade de carregamento/descarregamento de 7, 14 gf/s e 20 s de permanência em carga máxima. Onde foi observado o comportamento das seguintes propriedades segundo SHIMADZU Instruction Manual $[6,7]$ :

- HV - Dureza em escala Vickers.

- DHV-I e DHV-2 - durezas dinâmicas ligadas aos regimes elastoplástico e plástico de deformação, respectivamente.

- Hit - Resistência do material ao início da deformação permanente, descrita pela formulação presente na equação I.

$$
\text { Hit }=\frac{F_{\text {máx }}}{A p}
$$

- Cit - Fluência da indentação, que é a razão entre a diferença os valores da profundidade de indentação no início e fim do patamar de carga máxima pelo seu valor da profundidade de indentação no início do patamar.

\section{RESULTADOS E DISCUSSÃO}

Na Figura I são apresentadas as micrografias obtidas por MEV do aspecto microestrutural representativo da espessura da chapa na direção de laminação da amostra como recebida (Figura la, b), assim como das amostras 
deformadas por meio de laminação de LC (Figura Ic) e LA (Figura Id).

Ao se avaliar a morfologia presente na amostra CR (Figura la, b), nota-se para além da similaridade da microestrutura ao longo de toda espessura, aspectos distintos dos grãos. Onde alguns dos grãos são extremamente grosseiros e alongados, e com alinhamento na direção de laminação, intercalados por agrupamentos de grãos menos espessos, alguns alongados na direção de laminação e outros equiaxiais (Figura Ib), de acordo com a direção normal.

$O$ aspecto microestrutural da condição como recebida pode estar correlacionada com o processamento da liga, isto é, consequência da laminação a quente de uma liga com alta energia de falha de empilhamento sem ocorrência de transformação alotrópica no estado sólido. Ou seja, a estrutura de grãos apresentados retrata que a deformação a quente do material garantiu a este apenas recuperação dinâmica e estática da estrutura encruada [8].

Outro fator evidenciado na microestrutura da amostra CR é a existência de precipitados/inclusões dispersos, pela presença de pontos brancos (Figura Ib) e cavidades (Figura la - produzidas possivelmente pela dissolução da interface matriz-precipitado durante o polimento eletrolítico). Onde estes precipitados possivelmente são devidos ao tratamento final de precipitação, visando o superenvelhecimento (tratamento T735I) da condição como recebida.

No que diz respeito as microestruturas desenvolvidas nas duas rotas de laminação empregues no presente trabalho, são observados grãos alongados com aspecto de encruamento em ambos os processos (LC e LA - Figura Ic,d), exceto por grupamentos de grãos com ondulações na microestrutura resultante da LC.
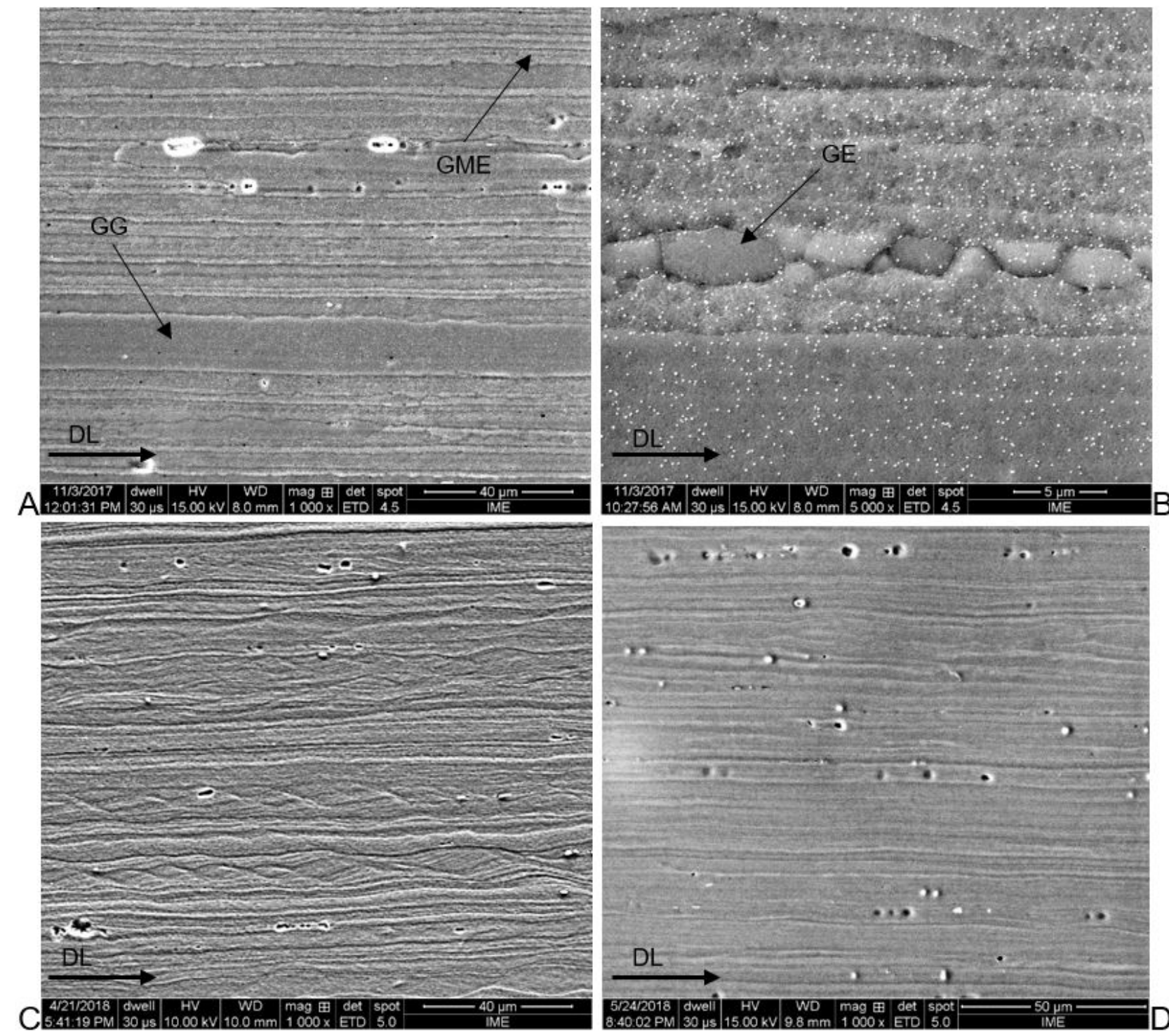

Figura I. Aspecto microestrutural a meia espessura avaliado por MEV. A, B: amostra CR, C: amostra LC; D: amostra LA. DL: direção de laminação; GG: grãos grosseiros; GME: grãos menos espessos e GE: grãos equiaxiais. 
Na Figura 2 são apresentados os resultados obtidos por ultramicrodureza instrumentada, associada a média das medidas em pontos distintos ao longo da espessura e seus respectivos desvios padrões.

Os resultados das durezas Vickers (HV), dinâmicas (DHV-I e DHV-2) e da indentação (Hit), e da fluência da indentação (Cit) apresentam comportamento distinto em relação a comparação entre as amostras (CR, LC e LA). Sendo mais evidentes os valores de desvio padrão, em todas as amostras, para a dureza DHV-2, possivelmente atribuído ao fato de suas medidas serem calculadas em função da profundidade de indentação somente devida a componente plástica de deformação $[6,7]$. O que deve ser fortemente influenciado pela microestrutura presente na região ao redor de cada indentação.

Observa-se um significativo incremento nos valores médios das durezas DHV-I e Hit para as amostras laminadas (LC e LA) em relação a como recebida (CR), porém o valor médio da Hit para a LA é ligeiramente superior a da LC e o inverso em relação ao valor médio de DHV-I. Enquanto o desvio padrão destas propriedades só é significativo para amostra LC. A justificativa para estes comportamentos deve estar associada a: (i) valores inferiores para CR devido a sua condição microestrutural de grãos mais grosseiros (Figura la) que oferecem uma menor resistência a indentação [9]; (ii) valores de LC próximos de LA, mas com desvio padrão superior, provavelmente é devido a refinamento microestrutural com evidências de aspecto heterogêneo ao longo da espessura na microestrutra em questão, pela presença de grão finos encruados alguns paralelos a direção de laminação e outros ondulados (Figura Ic).
Por outro lado, HV e DHV-2 apresentam andamento similar, em termos de média, mas com destaque ao significativo desvio padrão dos resutados de DHV-2, onde os valores médios e o desvio padrão da amostra LC são superiores aos de CR e LA, que apresentam valores próximos. Estes comportamentos podem ser justificados pelo significado físico associado ao cálculo destas propriedades [6,7]. Pois as durezas HV e DHV-2 (dinâmica plástica) são frutos da resistência mecânica que o material exerce em oposição a penetração do indentador (na carga máxima), sem levar em consideração a deformação elástica durante o processo de penetração do indentador. Enquanto as durezas DHV-I (dinâmica elastoplástica) e Hit (resistência a indentação para deformação permanente) se relacionam com a deformação elástica e plástica presente durante o processo de indentação. Deste modo, justifica-se o fato da amostra CR apresentar valores inferiores para as durezas DHV-I e Hit, em função da sua microestrutura contendo grãos grosseiros resultando em uma menor resistência ao início da deformação plástica e deformação a carga máxima. Além disso, o refinamento microestrutural promovido por ambas as rotas de laminação resultou em um aumento na resistência mecânica da liga submetida a estes processos [9].

Confrontando os valores médios da propriedade Cit (fluência da indentação) com as durezas DHV-I e Hit, observa-se comportamento oposto, pois os valores médios da amostra CR são superiores para esta primeira propriedade e inferiores e próximos para as amostras LC e LA. Tomando em consideração que o valor de Cit aumenta em função do incremento da profundidade de indentação ao longo do patamar da carga máxima. Podendo assim justificar o valor superior de Cit da amostra CR pela sua microestrutura

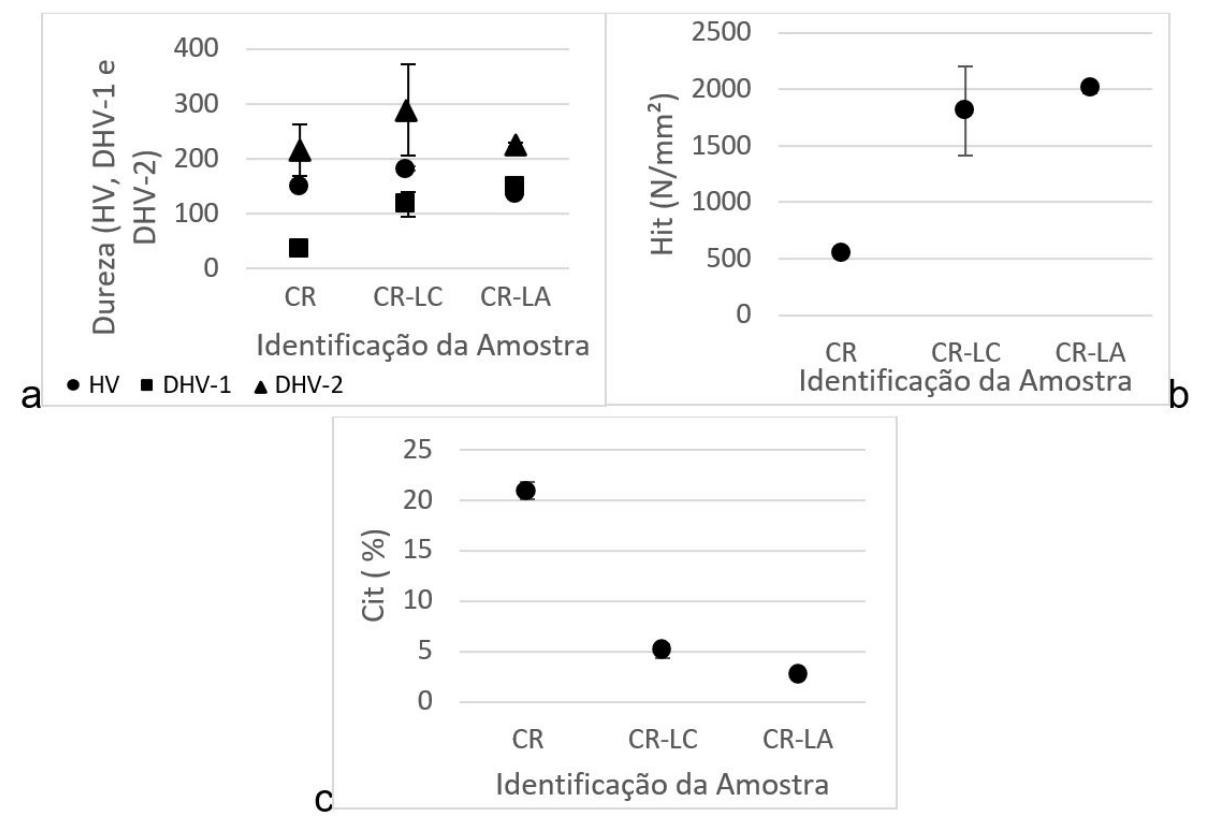

Figura 2. Propriedades mecânicas medidas por ultramicrodureza instrumentada: (a) HV, DHVI e DHV2; (b) Hit; e (c) Cit. 
contendo grãos grosseiros e valores próximos para as laminadas pelo seu refinamento microestrutural.

Pelos resultados analisados é clara a relação do encruamento promovido pelas rotas de laminação no aumento da resistência ao início e progresso da penetração do indentador durante aplicação da carga até a carga máxima. Como também, pelo fato do valor baixo de carga máxima adotado (50 gf), é notória a influência da microestrutural local nos resultados das propriedades analisadas. Sendo assim, um indicador quanto ao grau de homogeneidade microestrutural atingido associado ao nível de endurecimento alcançado.

\section{CONCLUSÕES}

Baseados na análise microestrutural e mecânica via ultramicrodureza instrumentada em distintas regiões ao longo da espessura, ao se comparar as amostras LC, LA e CR, o processo de laminação assimétrica (LA), diferentemente do que se era esperado, mostrou-se dentre as técnicas estudadas neste presente trabalho, a com maior capacidade de estabelecer uma homogeneidade microestrutural e de endurecimento no material ao longo da espessura. Indicando que a diferença de diâmetros entre os cilindros de trabalho não foi suficiente para impor assimetria microestrutural e mecânica.

\section{Agradecimentos}

Os autores agradecem à CAPES (bolsas de mestrado e doutorado) e ao CNPq (bolsa de doutorado T. G. Souza). Ao prof. Ladário da Silva que possibilitou o uso do equipamento de ultramicrodureza instrumentada, do Laboratório Multiusuário de Caracterização de Materiais do Instituto de Ciências Exatas (ICEx) da Universidade Federal Fluminense (UFF) de Volta Redonda - RJ. Ao prof. Neil de Medeiros da UFF pelo mancal do laminador.

\section{REFERÊNCIAS}

I Dursun T, Soutis C. Recent developments in advanced aircraft aluminium alloys. Materials \& Design. 20I4;56:86287I.

2 Hailang YU, Cheng LU, Kiet T, Xianghua L, Yong S, et al. Asymmetric cryorolling for fabrication of nanostructural aluminum sheets. Scientific Reports. 2012;2:772.

3 Magalhães DCC, Ferrante M, Sordi VL. Estudo comparativo entre laminação convencional e laminação assimétrica da liga AA606I: efeitos na microestrutura e propriedades mecânicas. In: Anais do $22^{\circ}$ Congresso Brasileiro de Engenharia e Ciência dos Materiaist; 2016 Novembro 6- I2; Natal, Brasil. São Paulo: Metallum; 2016. p. 5004-5016.

4 Casanova AMB, Diniz SB, Silva CS, Souza TG, Brandão LPM, Paula AS. Comparativo da propriedade HV entre amostras laminadas convencionalmente e por meio de laminação assimétrica. In: Anais da ABM Week $2017 ; 2017$ Novembro 2-6; São Paulo, Brasil. São Paulo: ABM; 2017. p. 3067-3075.

5 Society of Automotive Engineers. AMS4202D: aerospace material specification - aluminium alloy plate 5.Zn-2.2MgI.6Cu-0.22 Cr (7475-T735 I ) - solution heat treated, stress relieved by streching, and precipitation heat treated. United States: SAE International. (Issued 1978-07; Revised 2005-07; Reaffirmed 2012-03).

6 Shimadzu. DUH-2 I I/DUH-2 I IS instruction manual: dynamic ultra-micro hardness tester. Kyoto: Shimadzu Corporation; 2009.

7 Fischer-Cripps AC. The IBIS handbook of nanoindentation. Forestville: Fischer-Cripps Laboratories Pty Ltd; 2005.

8 Padilha AF, Siciliano JRF. Encruamento, recristalização, crescimento de grão e textura. 3. ed. rev. ampl. São Paulo: ABM Livros; 2005.

9 Reed-Hill, RE. Princípios de metalurgia física. 2. ed. Rio de Janeiro: Guanabara Dois; 1982.

Recebido em: 20 Nov. 2017

Aceito em: 26 Jul. 2018 